\title{
Management of Postoperative Neurosurgical Patients
}

\author{
Nidhi B. Panda ${ }^{1}$ Shalvi Mahajan ${ }^{2}$ Rajeev Chauhan ${ }^{3}$ \\ ${ }^{1}$ Division of Neuroanaesthesia, Department of Anaesthesia and \\ Intensive Care, Post Graduate Institute of Medical Education \& \\ Research (PGIMER), Chandigarh, Punjab, India \\ ${ }^{2}$ Department of Anesthesia and Intensive Care, Fortis Hospital, \\ Mohali, Punjab, India \\ ${ }^{3}$ Department of Anaesthesia and Intensive Care, Post Graduate \\ Institute of Medical Education \& Research (PGIMER), Chandigarh, \\ Punjab, India
}

J Neuroanaesthesiol Crit Care 2019;6:80-86

\begin{abstract}
Address for correspondence Nidhi B. Panda, MBBS, MD Anesthesia, Division of Neuroanaesthesia, Department of Anaesthesia and Intensive Care, Post Graduate Institute of Medical Education \& Research (PGIMER), Chandigarh 160012, Punjab, India (e-mail: nidhibp@gmail.com).
\end{abstract}

\begin{abstract}
Keywords

- neurocritical care

- aneurysmal subarachnoid hemorrhage

- traumatic brain injury

- neurosurgery

Neurosurgical patients are a special subset of patients requiring postoperative care. Challenging neurosurgical disease processes, advanced surgical techniques, and unique individual patient requirements advocate the need for meticulous postoperative care to ensure safe transition toward recovery. Timely detection of systemic and neurological changes allows early diagnostic and therapeutic interventions. The mainstay of postoperative care revolves around airway, maintenance of hemodynamics, sedation, analgesia, nutrition, fluid management, and management of disease-specific complications. In addition to standard monitoring, multimodal neuromonitoring should be used in neurosurgical patients. Hence, four key elements in the postoperative management of neurosurgical patients involve profound insight, rapid response, good communication skills, and team collaboration.
\end{abstract}

\section{Introduction}

The concept of dedicated neurocritical care (NCC) unit was conceived late back in the 1900 s. ${ }^{1}$ NCC is a specialized critical care unit staffed by a team comprising neurosurgeons, neurointensivists, neurophysiologists, specialized nursing staff, physiotherapists (respiratory and rehabilitation), and nutritionists. Studies have reported positive results and better outcomes in patients managed postoperatively in NCC with aneurysmal subarachnoid hemorrhage (aSAH), stroke, traumatic brain injury (TBI), and spine surgeries in terms of intensive care unit (ICU) stay and hospital discharge. ${ }^{2}$

\section{Criteria for Admission of Postoperative Neurosurgical Patients in NCC Unit}

Postoperative neurosurgical patients can be grouped into elective and emergency categories based on their presentation. Admission in the NCC unit following neurosurgical procedure is dictated by patient's neurologic and medical condition. Certain preoperative and intraoperative factors may determine the need for postoperative ICU care, which include preoperative altered consciousness, prolonged duration of

received

February 5, 2019

accepted after revision

April 5, 2019

published online

June 6, 2019 surgery ( $>6$ hours) and major blood loss with hemodynamic instability, extensive posterior fossa surgery involving cranial nerves and brain stem, arteriovenous malformation (AVM) resection with chances of postoperative malignant edema, and vascular surgery with significant brain ischemia.

Although the incidence of perioperative mortality is less than $2 \%$, medical and neurologic complications may occur in the postoperative period in up to $30 \%$ of cases. Therefore, perioperative complications must be identified and managed before the patient's neurologic and medical status is irreversibly compromised. Various postoperative complications following brain surgery require admission in the NCC unit (-Table 1).

\section{Assessment of Postoperative Neurosurgical Patients}

Standardized systemic examination, neurological assessment, and neuromonitoring are valuable to recognize early deterioration in the postoperative period ( - Fig. 1).

General physical examination and close monitoring of electrocardiogram (ECG), peripheral capillary oxygen saturation $\left(\mathrm{SpO}_{2}\right)$, blood pressure (invasive and noninvasive), temperature, central venous pressure along with

Copyright $\odot 2019$ Indian Society of Neuroanaesthesiology and Critical Care
License terms

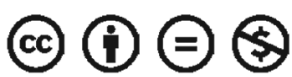

10.1055/s-0039-1689055 ISSN 2348-0548. 
Table 1 Postoperative complications in neurosurgical patients

\begin{tabular}{|l|l|}
\hline Systemic & Neurological \\
\hline Hypoxia & Cerebral edema \\
\hline Hypotension/hypertension & Hematoma/rebleeding \\
\hline Hypocapnia/hypercapnia & Hydrocephalus \\
\hline Hyperthermia & Pneumocephalus \\
\hline Hyperglycemia/hypoglycemia & Seizures \\
\hline Hyponatremia/hypernatremia & $\begin{array}{l}\text { Delayed cerebral } \\
\text { ischemia }\end{array}$ \\
\hline Coagulation disorders & $\begin{array}{l}\text { Central nervous system } \\
\text { infections }\end{array}$ \\
\hline Thromboembolic events (DVT/PE) & \\
\hline Anemia & \\
\hline
\end{tabular}

Abbreviations: DVT, deep vein thrombosis; PE, pulmonary embolism.

measurement of serum electrolytes, osmolality, blood glucose, and arterial blood gases are necessary. Priority management in NCC is prompt stabilization of all vital parameters to prevent secondary neurological insult.

Neurological examination involves assessment of general responsiveness (by Glasgow Coma Score [GCS], full outline of unresponsiveness [FOUR] score), pupils, cranial nerve function, reflexes, and motor power. The functional scoring systems are indispensable since they help in assessing neurological status quantitatively and comparing neurological status with previous assessment. The most commonly used score in neurological patients is GCS. ${ }^{3}$ The FOUR score was introduced by Dr. Eelco F. M. Wijdicks for the assessment of patients with impaired level of consciousness. It offers information about brainstem reflexes (pupil, corneal, and cough), respiration (breathing patterns, respiratory drive while on ventilator), visual tracking (eye component), and motor response. ${ }^{4}$ It does not take into account verbal component as in GCS.

Neuromonitoring: The concept of modern multimodal neuromonitoring has shifted from rigid physiological target-based approach to a more patient-specific approach. Multimodal neuromonitoring helps in early identification of pathophysiological indicators of secondary brain injury. Hence, it is imperative for the neurointensivist to intervene at the earliest before permanent neural cell death occurs. Intracranial pressure (ICP), cerebral blood flow, brain oxygenation, electrophysiology, and neuroimaging are indispensable components of neuromonitoring.

In many intracranial events, raised ICP and low cerebral perfusion pressure (CPP) cause cerebral ischemia. ICP monitoring is an important component of multimodal neuromonitoring and helps in maintaining CPP. There are both invasive and noninvasive monitoring techniques. The gold standard technique is intraventricular pressure monitoring, which has the added advantage of draining cerebrospinal fluid (CSF) and administering intraventricular medications. ${ }^{5}$ The commonly used noninvasive method of ICP monitoring is measurement of optic nerve sheath diameter using ultrasound. ${ }^{6}$

Cerebral blood flow can be monitored noninvasively in the postoperative period using transcranial Doppler (TCD).
It measures cerebral blood flow velocities and other derived indices (pulsatility index) which help in the diagnosis of cerebral vasospasm and hyperemia.7 TCD can also be used to assess pressure autoregulation, cerebrovascular carbon dioxide reactivity, and noninvasive estimation of ICP.

Cerebral oxygenation can be assessed using jugular venous oximetry, cerebral oximetry, and brain tissue oxygenation. ${ }^{8}$ Jugular venous oximetry is an invasive method to measure oxygen saturation of the venous blood at the jugular bulb and provides information of global oxygen extraction of the cerebral tissues. Cerebral oximetry is a noninvasive continuous technique to measure regional cerebral oxygenation using near-infrared spectroscopic technique. Brain tissue oxygen monitoring represents oxygen available at focal cerebral mitochondrial level for oxidative phosphorylation.

Cerebral microdialysis allows monitoring of metabolic milieu of brain tissue (glucose, lactate, pyruvate, glycerol, glutamate, cytokines, neurotrophic, and other neuronal markers). However, it is not widely used for clinical purposes. ${ }^{9}$

\section{General Management Goals of Postoperative Patients in Neurocritical Care Units}

Postoperative complications following neurosurgical procedures are mainly associated with the basic pathology of the disease or the sequel of intraoperative surgical and anesthetic techniques. Inability to rescue a patient from potentially reversible complications is an important reason of perioperative morbidity and mortality ( - Fig. 2 ).

\section{POSTOPERATIVE MONITORING}
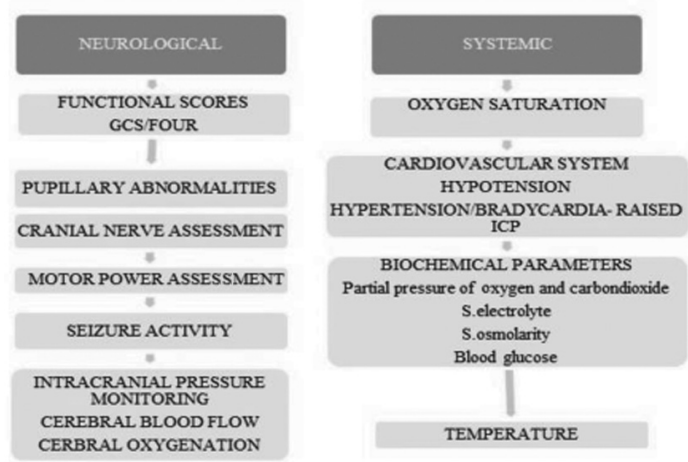

Fig. 1 Postoperative monitoring in neurosurgical patients. FOUR, full outline of unresponsiveness; GCS, Glasgow Coma Score; ICP, intracranial pressure.

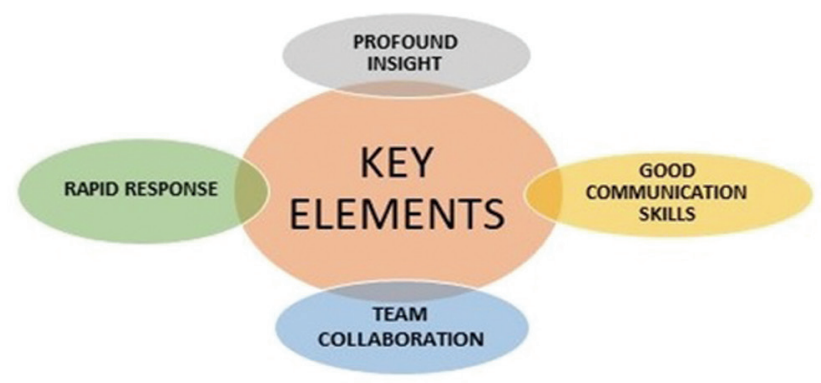

Fig. 2 Key elements in the management of postoperative neurosurgical patients. 


\section{Airway Management and Ventilation}

In patients with preoperative GCS $<8$, lower cranial nerve dysfunction, and weak gag and cough reflexes, the airway may need to be secured. Airway obstruction may be seen in the postoperative period in acromegaly patients (due to macroglossia and obstructive sleep apnea). Postoperative patients following anterior cervical spine surgery or carotid endarterectomy may also present with airway obstruction due to edema or hematoma. Irregular breathing pattern is common postoperatively in patients with posterior fossa or brain stem lesions. Acute brain insult may cause neurogenic pulmonary edema (NPE). ${ }^{10}$

The benefit of securing airway and elective ventilation is to avoid hypoxia and hypercapnia, both of which aggravate neuronal injury in postoperative period. Hence, arterial oxygen and carbon dioxide levels must be monitored regularly. A single episode of hypoxemia $\left(\mathrm{SpO}_{2}\right.$ $<90 \%$, partial pressure of oxygen $\left[\mathrm{PaO}_{2}\right]<60 \mathrm{~mm} \mathrm{Hg}$ ) increases the chance of adverse outcome by $40 \%{ }^{11}$

Partial pressure of carbon dioxide $\left(\mathrm{PaCO}_{2}\right)$ is a powerful modulator of cerebrovascular resistance. Ventilation aims to maintain $\mathrm{PaCO}_{2}$ levels within the normocapnic range. Hypoventilation may lead to cerebral hyperemia with increase in the cerebral blood volume and rise in ICP, while hyperventilation increases the risk of vasoconstriction and tissue hypoxia. In the management of intracranial hypertension, hyperventilation strategies are useful to decrease ICP. Hyperventilation is only useful as a short-term strategy as pH of CSF gradually normalizes over 6 to 8 hours as a result of extrusion of bicarbonate. Volume-controlled ventilatory modes may be better for minimizing variations in $\mathrm{PaCO}_{2}$ levels. Positive end expiratory pressure (PEEP) of 5 to $8 \mathrm{~cm} \mathrm{H}_{2} \mathrm{O}$ can be administered safely to prevent atelectasis. Patients with concurrent acute pulmonary injury need protective pulmonary ventilation strategy with small tidal volume and PEEP (up to $15 \mathrm{~cm} \mathrm{H}_{2} \mathrm{O}$ ). ${ }^{12}$ In patients with normal respiratory compliance, high PEEP increases intrathoracic pressure and jugular venous pressure, and decreases venous return (cardiac output) which can lead to decrease in CPP if cerebral autoregulation is impaired. ${ }^{13}$ But in patients with low respiratory compliance, significant effects of PEEP are not seen on systemic and cerebral hemodynamics. ${ }^{14}$

Head up position: In neurosurgery patients, it is advantageous to keep patients in slight $\left(15-30^{\circ}\right)$ head up position so as to promote venous drainage and decrease the risk of increase in ICP. Also, it helps in reducing risk of aspiration.

\section{Cardiovascular Changes and Management}

Postoperative hypertension can precipitate cerebral edema, intracranial hemorrhage as well as extracranial hemorrhage. Therefore, strict control of blood pressure is essential in hypertensive patients who have undergone intracranial vascular surgery. Hypertension may be a part of Cushing reflex, or consequent to acute pain or hypercarbia. It is reasonable to use invasive blood pressure monitoring in such conditions in the postoperative period.
Postoperative hypotension should be adequately treated with fluids and vasopressors. Hypotension causes a decrease in the cerebral blood flow and CPP, which results in reflex vasodilatation, increased cerebral blood volume, and high ICP. Both increase in ICP and decrease in CPP in the postoperative period are disastrous in terms of neurological outcome.

Cardiac changes are well-known after acute brain insult. ${ }^{15}$ Meticulous monitoring of ECG for arrhythmias and myocardial ischemia should be done. Biomarkers of cardiac injury (cardiac troponin I, creatine phosphokinase-myocardial fraction [CK-MB], and serum B-type natriuretic peptide) can be elevated after neurosurgeries. Cardiac troponin I is more sensitive for the detection of left ventricle dysfunction than CK-MB and high levels are associated with poor functional outcome in survivors. Partial or even complete resolution of cardiac changes follows decrease in ICP. Echocardiography can show regional wall motion abnormalities, transient left ventricular wall motion abnormalities involving apical or mid-ventricular segments that extend beyond single epicardial coronary artery distribution in SAH..$^{16}$ Increased sympathetic activity seen in paroxysmal sympathetic hyperactivity has been associated with poor outcomes. ${ }^{17}$

\section{Postoperative Analgesia and Postoperative Nausea and Vomiting}

Most of the patients experience moderate-to-severe pain following major intracranial surgery for the first 48 hours. Patients undergoing foramen magnum decompression and acoustic neuroma resection are at a high risk to develop severe pain in the postoperative period. ${ }^{18}$ Adequate pain control ensures patient's comfort, facilitates accurate neurological assessment, prevents increase in blood pressure, and allows early recovery. Multimodal treatment with paracetamol, opioids, local anesthetic infiltration, and scalp block provides adequate analgesia in the postoperative period.

The overall incidence of postoperative nausea and vomiting (PONV) following craniotomies is around $50 \% .^{19}$ PONV is common, especially after infratentorial surgery (foramen magnum decompression and acoustic neuroma resection).20 Vomiting causes a rise in ICP due to systemic hypertension and increase in abdominal and intrathoracic pressure. Medications such as ondansetron, dexamethasone, cyclizine, and even hyoscine patch can be used to prevent and treat PONV.

\section{Postoperative Fluid and Electrolyte Disturbances}

Neurosurgical patients often exhibitchanges in intravascularvolume owing to use of potent diuretics, prolonged fasting, vomiting (raised ICP), hemorrhage, and electrolyte disturbances. The movement of water between brains extracellular area and vessels is primarily driven by osmotic gradient. Use of hypo-osmolar fluids such as $5 \%$ dextrose and $0.45 \%$ saline potentiate brain edema, while hyperosmolar agents such as mannitol, glycer$\mathrm{ol}$, and hypertonic saline decrease cerebral edema. Hence in postoperative period, fluid status should be monitored using dynamic hemodynamic parameters, and isotonic crystalloids should be used to maintain euvolemia. 
Sodium disturbance, either hypo- or hypernatremia, is the most common electrolyte disturbance in neurosurgical patients. ${ }^{21}$ Intracranial diseases involving surgeries of the hypothalamus, pituitary, or cerebral trauma may cause diabetes insipidus (DI). It is characterized by polyuria, hypernatremia, increased plasma osmolarity, decreased urine specific gravity and osmolarity. Management involves fluid and antidiuretic hormone (ADH) supplementation. Following brain insult (trauma or SAH), patients may suffer from syndrome of inappropriate ADH secretion (SIADH) or cerebral salt wasting syndrome (CSWS). SIADH is a volume expanded state characterized by oliguria, weight gain, low serum sodium, and high urine sodium (>25 mmol/L), whereas CSWS manifests as hyponatremia, dehydration, and high urine sodium (>50 $\mathrm{mmol} / \mathrm{L}$ ). In both conditions, hyponatremia is seen but the management is entirely different. Treatment of SIADH depends on fluid restriction ( $<1 \mathrm{~L} / 24 \mathrm{~h}$ ), sodium supplementation (3\% hypertonic saline), while CSWS requires isotonic sodium solutions to re-establish circulating volume.

Postoperative venous thromboembolism: It includes deep vein thrombosis (DVT) and pulmonary embolism. Cumulative incidence varies from 20 to $50 \%$ after neurosurgical procedures. Dehydration (use of mannitol/furosemide), immobility, paralysis, TBI (release of procoagulant), stroke, and certain brain lesions (meningioma) are considered as risk factors for the development of thromboembolism. Prophylaxis includes limb physiotherapy, mechanical devices (intermittent pneumatic devices, graduated compression stockings), and pharmacological anticoagulants (unfractionated or low molecular weight heparin). Pharmacological prophylaxis is usually started after 48 hours of surgery when risk for bleeding or increase in size of hematoma is low.

Hormone replacement: In pituitary surgery almost all patients receive corticosteroid coverage until testing indicates an intact pituitary-adrenal axis. Thyroid hormone replacement is reserved for patients with preoperative hypothyroidism.

Antibiotics: Postoperative antibiotic prophylaxis is usually not recommended except in cases where foreign materials are implanted (such as intracranial electrodes in deep brain stimulation or epilepsy surgery), or continuous ICP monitoring is done using invasive methods. Similarly, it is indicated in cases of contaminated wounds such as in TBI, or when the risk of introducing infection is high like in CSF leak.

Temperature management: Euthermia should be maintained in the postoperative period. Hypothermia in cases of prolonged surgery and exposure to cold environment (catheterization laboratory or magnetic resonance imaging suit) delays recovery and increases the risk of developing postoperative infections. Postoperative shivering increases body oxygen consumption by up to 300 to $400 \% .^{22}$ Hyperthermia in neurosurgical cases is equally detrimental.

Nutrition status: Feeding should be started at the earliest in the postoperative period, either by oral, nasogastric, or transgastric route, along with prophylaxis for stress ulcer.
Blood glycemic control: Extreme levels of blood glucose influence neurological outcome negatively. Glycemic control is affected in patients who receive corticosteroids as a part of hormone replacement therapy or as a decongestive measure to decrease vasogenic edema. Blood glucose management in neurosurgery tends toward moderate plasma glucose level goals (140-180 mg/dL). The exact mechanism how hyperglycemia exacerbates ischemic neuronal injury is not clearly known but oxidative stress at cellular level is implicated. Oxidative stress due to production of advanced glycosylated end-products, polysols, and hexosans lead to elevation of inflammatory cytokines and suppression of immune system. Also, hyperglycemia is associated with release of excitatory neurotransmitter glutamate in the neocortex. During ischemia, neuronal glucose (stored in hyperglycemic phase) results in increased intracellular lactate. The intracellular lactate is neurotoxic and is believed to enhance neuronal injury.

\section{Common Intracranial Postoperative Complications Seen in NCC Units}

Cerebral edema: Risk factors causing edema may include preoperative cerebral edema, prolonged duration of surgery, excessive brain retraction, following AVM resection and redo surgeries. Treatment involves use of dexamethasone to decrease vasogenic edema, use of osmotic agents, adequate analgesia/sedation, normoventilation to mild hyperventilation, and use of iso-osmolar to mild hyperosmolar fluids.

Intracranial hemorrhage: Postoperative risk of intracranial hemorrhage increases in patients with hypertension, coagulopathy and poor surgical homeostasis. Once suspected, noncontrast computed tomography (CT) scan is indicated and depending on the site and size of the hematoma, emergent evacuation may be required. Mannitol and reintubation may be required to stabilize the patient's condition.

Hydrocephalus: After intraventricular bleed or in posterior fossa surgery, there are chances of development of hydrocephalus. It may require external ventricular drain insertion in the postoperative period.

Seizures: Cortical pathology has a higher chance of developing seizures. Patients with epilepsy, brain tumor, aneurysmalSAH and intracranial hemorrhage are prone to developing seizures. Postoperative drop in the functional neurological score could be due to convulsive or nonconvulsive seizures. Routine seizure prophylaxis is generally not recommended for most intra-cranial tumors unless there is evidence of seizures. In postoperative seizures, phenytoin or levetiracetam can be used. Phenytoin is used with a loading dose 10 to $20 \mathrm{mg}$ phenytoin equivalents (PE)/kg over 30 minutes, maximum rate $150 \mathrm{mg}$ $\mathrm{PE} /$ minute intravenously, no faster than $50 \mathrm{mg} /$ minute in an adult; followed by infusion of 5 to $7 \mathrm{mg} / \mathrm{kg} /$ day. Fosphenytoin is given at a loading dose of 15 to $20 \mathrm{mg} / \mathrm{kg}$ intravenously, no faster than 100 to $150 \mathrm{mg} /$ minute, followed by an infusion of 4 to $6 \mathrm{PE} / \mathrm{kg} /$ day. Levetiracetam is used in doses of $1000 \mathrm{mg} /$ day to $3000 \mathrm{mg} /$ day in two divided dosages. 
Cerebrospinal fluid leak: CSF leak is commonly seen after trans-sphenoidal pituitary surgery, TBI, or incisional CSF leak following posterior fossa surgery. Clinical symptoms include leakage of clear, nonmucoid, watery fluid from the nose, ear, or suture site which has positional dependency. Sometimes, it also presents as a salty postnasal drip. Further testing of clear fluid mixed with blood or nasal discharge on a filter paper shows double ring or halo or target sign. The handkerchief test differentiates nasal discharge (unclear, sticky due to mucin secretion) and CSF (clear and nonsticky). Glucose level in CSF > $30 \mathrm{mg} / \mathrm{dL}$ indicates CSF leak. Beta-2 transferrin and beta-trace proteins are specific markers of CSF.CSF rhinorrhea predisposes patients to meningitis, intracranial hypotension and leads to prolonged hospital stay. Early detection and repair of the leak are essential. Incisional CSF leak requires resuturing of the wound, concomitant CSF lumbar drainage, and administration of antibiotics.

Pneumocephalus: Patients operated in sitting or semi-sitting positions are at risk of developing pneumocephalus. Pneumocephalus may also develop in other surgeries such as trans-sphenoidal surgery or chronic subdural hematoma evacuation. Large amount of air trapping results in altered sensorium, seizures and coma. Tension pneumocephalus requires urgent decompression.

Cranial nerve deficits: Cranial nerve deficits are common in posterior fossa surgery or skull base surgery. Injury can cause impaired bulbar function, which requires reconsideration of airway protection due to the possibility of aspiration pneumonia.

Meningitis: Meningitis can be seen in postoperative cases of TBI, use of intraoperative electrodes, following CSF leak, or due to failure to use sterile surgical techniques. It is important to identify meningitis and promptly initiate treatment.

\section{Specific Neurological Conditions}

Supratentorial surgery: Postoperative hematoma, cerebral edema, seizures, postcraniotomy pain, and PONV are common complications following supratentorial surgeries. Tumor bed hematoma due to incomplete hemostasis, subdural or epidural hematoma are not uncommon. Prevention of hypertension or excessive coughing and bucking during emergence is important. Management involves urgent noncontrast CT head, medical treatment to decrease raised ICP, hematoma evacuation, and decompressive craniectomy. Delayed hydrocephalus may occur after resection of supratentorial malignant gliomas and should be considered in cases of initial improvement followed by deterioration of consciousness. Shunting of CSF in cases of communicating hydrocephalus yields satisfactory results.

Pituitary surgery: Endocrine complications following pituitary surgery include hypocortisolism, hypothyroidism, and DI. Treatment of DI consists of hydration and hormonal supplementation. The volume and type of intravenous fluids are guided by urine volume, serum electrolytes, and osmolality. Free water $\left(\mathrm{H}_{2} \mathrm{O}\right)$ deficit can be estimated using the formula: Free $\mathrm{H}_{2} \mathrm{O}$ deficit $(\mathrm{L})=($ serum Na-140 $) \times$ body weight $(\mathrm{kg}) \times 0.6 / 140$. Free water deficit can be replaced by oral fluids. If it has to be replaced intravenously, hypotonic solutions such as $0.45 \%$ sodium chloride $(\mathrm{NaCl})$ can be given. Insulin and potassium supplementation might be required when dextrose-containing fluids are used, and when corticosteroids are administered concomitantly. When hormonal replacement is required, 1-deamino-8-d-arginine vasopressin, a synthetic analog of the natural hormone arginine vasopressin, can be given intravenously, subcutaneously, orally, or intranasally. The latter might not be feasible after transnasal trans-sphenoidal pituitary surgery. The usual intravenous or subcutaneous dose is $0.3 \mu \mathrm{g} / \mathrm{kg} /$ day in two divided doses. The dose of oral medication is 0.05 to $1.2 \mathrm{mg} /$ day at once or divided into two or three doses. The intranasal dose is 10 to $40 \mu \mathrm{g} /$ day in one to three doses.

Vision loss in the immediate postoperative period can be due to hematoma formation compressing on the optic nerve or optic chiasma, which requires immediate surgical evacuation, while delayed loss can be due to cerebral edema, which requires supportive NCC. A thorough evaluation of postoperative rhinorrhea for CSF leak is important. CSF leak increases risk of meningitis and secondary neurological injury. Treatment options include lumbar drainage of CSF and surgical repair of the defect.

Infratentorial surgery: Patients undergoing posterior fossa surgeries may have delayed emergence due to tension pneumocephalus, intracranial hemorrhage, symptomatic venous air embolism, and damage to the brain stem. Elective postoperative ventilation is required in cases of bulbar involvement and extensive intraoperative dissection near the brain stem (central respiratory depression). Injury to lower cranial nerves (IX, X, and XII) may compromise the patient's ability to maintain a patent protected airway due to difficulty in swallowing and clearing secretions. Injury to ophthalmic division of the trigeminal nerve may impair protective reflexes of the cornea and require external protection with an eye patch. Postoperative visual loss subsequent to surgery in prone position is another devastating complication. Severe postoperative pain due to extensive muscle dissection and muscle spasm needs adequate analgesia to prevent development of chronic pain syndromes. Close proximity of the surgical site to vomiting centers and use of opioids for postoperative pain relief increase the risk of PONV and require multimodal antiemetic therapy.

Subarachnoid hemorrhage: Postoperative period following surgical clipping in aSAH is crucial. Life-threatening vasospasm is usually seen from 3 to 14 days after SAH. It is clinically identified as decrease in consciousness, disorientation, and appearance of new focal neurological deficits. Clinical vasospasm is seen in 30\% cases, while radiographic vasospasm is seen in $70 \%$ cases.Different parameters that aid in the diagnosis of vasospasm include flow velocity measurement by TCD (increased flow velocity $>120 \mathrm{~cm} / \mathrm{s}$ or Lindegaard ratio $>3$ ), decreased amplitude, and decreased alpha activity in electroencephalography. Digital subtraction angiography offers diagnostic as well as therapeutic (intra-arterial nimodipine/milrinone administration) modality. Numerous treatment options are available but approved treatment involves use of oral nimodipine, maintenance of euvolemic 
status, and maintenance of mild hypertension. ${ }^{23}$ Fluid and electrolyte disturbances are common in aSAH. Up to $40 \%$ patients experience hyponatremia either due to CSWS or SIADH. Management of CSWS and SIADH has been described earlier. Acute hydrocephalus can occur in $20 \%$ of aSAH. Patients may have persistent headache, loss of upward gaze, and cognitive impairment, and may require CSF drainage.

Traumatic brain injury: The systemic sequelae of head trauma frequently become apparent in the postoperative period. These include cardiac arrhythmias, disseminated intravascular coagulation, adult respiratory distress syndrome, NPE, DI, SIADH, hyperglycemic hyperosmolar nonketotic coma, and gastrointestinal stress ulcers and hemorrhage. Moderate-to-severe head injury patients require intensive care for prevention of secondary injury, early detection, and management of neurological deterioration and management of ICP.

NPE is a fulminant form of pulmonary edema, which can progress rapidly (within hours to days) toward either resolution or death. Treatment is aimed at reducing ICP, reducing sympathetic hyperactivity, mainly with pharmacological agents, providing respiratory supportive care, and inotropic therapy as needed.

SIADH treatment consists of water restriction, use of loop diuretics, and hypertonic saline. In mild cases, fluid restriction (1-1.5 $\mathrm{L} /$ day) is sufficient to correct hyponatremia. Furosemide may be added to treat hypervolemia. Hypertonic saline is usually reserved for a serum $\mathrm{Na}<120 \mathrm{mEq} / \mathrm{L}$. It is given in small amounts for a short time $(1-2 \mathrm{~mL} / \mathrm{kg} / \mathrm{h}$ for 2-3 h) after which serum sodium and osmolality are measured. During the acute phase of SIADH, urine output should be measured hourly, urine osmolality and specific gravity, and serum sodium and osmolality are measured every 6 to 8 hours. Serum sodium should be increased at a rate of not more than $0.5 \mathrm{mEq} / \mathrm{L} /$ hour or $12 \mathrm{mEq} / \mathrm{L} /$ day.

Brain Trauma Foundation guideline states ICP above $22 \mathrm{~mm} \mathrm{Hg}$ as threshold for treatment. The treatment of raised ICP follows stepwise pattern starting with head elevation $\left(30^{\circ}\right)$, normoventilation (or short period of hyperventilation), adequate sedation, analgesia, and muscle paralysis (to prevent coughing and bucking on ventilator and decreasing work of breathing). If ICP continues to be high ( $>25 \mathrm{~mm}$ $\mathrm{Hg}$ ), hyperosmolar therapy (mannitol/hypertonic saline) and ventriculostomy can be added to the previous treatment. Other options to decrease raised ICP include therapeutic hypothermia, decompressive craniectomy, and inducing barbiturate coma. Although hypothermia is neuroprotective, therapeutic hypothermia has not been found to improve functional neurological outcomes. ${ }^{24,25}$

Occlusive cerebrovascular surgery: Carotid sinus baroreceptor function impairment after carotid endarterectomy or angioplasty causes hypotension, hypertension, and dysrhythmias. Mild increase in blood pressure (up to $20 \%$ above baseline level) is acceptable but above this requires antihypertensive therapy. Major complications anticipated include stroke, myocardial infarction, and hyper-perfusion syndrome.
Arteriovenous malformation: Following AVM resection or obliteration, patients are at risk of developing complications similar to those that occur after aneurysm surgery (vasospasm, hydrocephalus, and seizures). In addition, these patients are at high risk of developing hyperemic complications leading to uncontrollable cerebral swelling and hemorrhage due to transient circulatory disturbance known as normal perfusion pressure breakthrough. This can manifest as headache, seizures, permanent neurological disability, and even death. Treatment strategies include fluid restriction, prevention of systemic hypertension, controlled hyperventilation, osmotic agents, and use of anesthetic agents to prevent raised ICP.

Interventional neuroradiological procedures: These patients should be kept in supine position till femoral sheath is in situ. Adequate control of blood pressure in postoperative period depends upon the type of cerebrovascular pathology. Contrast-induced nephropathy (CIN) is the third most common cause of hospital-acquired renal failure. The risk factors include diabetes mellitus, high dose of contrast, volume depletion, co-administration of nephrotoxic drugs, and pre-existing renal disease. To prevent renal complications, perioperative management should be aimed at maintaining normovolemia using intravenous saline hydration and the use of low osmolality contrast medium. The use of $\mathrm{N}$-acetylcysteine to decrease the risk of CIN is debatable. ${ }^{26}$ In view of current evidence, it is probably not indicated as the standard-of-care.

Spine surgery: Anterior cervical spine surgeries increase the risk of developing postoperative airway obstruction due to pharyngeal edema, wound hematoma, vocal palsy, dislodgment of bone graft, or fixation plate. Management involves emergency reintubation followed by hematoma evacuation. Postoperative pain management is an important aspect as it interferes with functional recovery following surgery (impaired ventilation in thoracic surgery, reduced early mobilization, increased risk of DVT, delayed bowel function, and increased risk of urinary infections). Multimodal pain management is an essential part of postoperative care of these patients. Prolonged spine surgeries along with immobilization and hypercoagulable states increase the risk of thromboembolic events in the postoperative period.

\section{Conclusion}

Outcome after neurosurgical procedure depends not only on surgical technique but also on postoperative care of these patients in specialized NCC units. The postoperative management of neurosurgical patients is a complex endeavor and it demands comprehensive knowledge of anatomical, physiological, and neurological perturbations. Careful regular assessment, multimodal neuromonitoring along with early detection, and management of any complication by an alert neurointensivist remain cornerstone of postoperative management. 


\section{Conflict of Interest}

None declared.

\section{References}

1 Lassen HC. A preliminary report on the 1952 epidemic of poliomyelitis in Copenhagen with special reference to the treatment of acute respiratory insufficiency. Lancet 1953;1(6749):37-41

2 Mirski MA, Chang CW, Cowan R. Impact of a neuroscience intensive care unit on neurosurgical patient outcomes and cost of care: evidence-based support for an intensivist-directed specialty ICU model of care. J Neurosurg Anesthesiol 2001;13(2):83-92

3 Teasdale G, Jennett B. Assessment of coma and impaired consciousness. A practical scale. Lancet 1974;2(7872):81-84

4 Wijdicks EF, Bamlet WR, Maramattom BV, Manno EM, McClelland RL. Validation of a new coma scale: the FOUR score. Ann Neurol 2005;58(4):585-593

5 Miller JD. Measuring ICP in patients: its value now and in the future? In: Hoff JT, Betz AL, eds. Intracranial Pressure VII. Berlin: Springer-Verlag; 1989:5-15

6 Rajajee V, Vanaman M, Fletcher JJ, Jacobs TL. Optic nerve ultrasound for the detection of raised intracranial pressure. Neurocrit Care 2011;15(3):506-515

7 Aaslid R, Markwalder TM, Nornes H. Noninvasive transcranial Doppler ultrasound recording of flow velocity in basal cerebral arteries. J Neurosurg 1982;57(6):769-774

8 Chieregato A, Calzolari F, Trasforini G, Targa L, Latronico N. Normal jugular bulb oxygen saturation. J Neurol Neurosurg Psychiatry 2003;74(6):784-786

9 Hillered L, Vespa PM, Hovda DA. Translational neurochemical research in acute human brain injury: the current status and potential future for cerebral microdialysis. J Neurotrauma 2005;22(1):3-41

10 Davison DL, Terek M, Chawla LS. Neurogenic pulmonary edema. Crit Care 2012;16(2):212

11 McHugh GS, Engel DC, Butcher I, et al. Prognostic value of secondary insults in traumatic brain injury: results from the IMPACT study. J Neurotrauma 2007;24(2):287-293

12 Davis DP. Early ventilation in traumatic brain injury. Resuscitation 2008;76(3):333-340

13 Muench E, Bauhuf $\mathrm{C}$, Roth $\mathrm{H}$, et al. Effects of positive end-expiratory pressure on regional cerebral blood flow, intracranial pressure, and brain tissue oxygenation. Crit Care Med 2005;33(10):2367-2372
14 Caricato A, Conti G, Della Corte F, et al. Effects of PEEP on the intracranial system of patients with head injury and subarachnoid hemorrhage: the role of respiratory system compliance. J Trauma 2005;58(3):571-576

15 Zygun D. Non-neurological organ dysfunction in neurocritical care: impact on outcome and etiological considerations. Curr Opin Crit Care 2005;11(2):139-143

16 Banki N, Kopelnik A, Tung P, et al. Prospective analysis of prevalence, distribution, and rate of recovery of left ventricular systolic dysfunction in patients with subarachnoid hemorrhage. J Neurosurg 2006;105(1):15-20

17 Meyfroidt G, Baguley IJ, Menon DK. Paroxysmal sympathetic hyperactivity: the storm after acute brain injury. Lancet Neurol 2017;16(9):721-729

18 Thibault M, Girard F, Moumdjian R, Chouinard P, Boudreault D, Ruel M. Craniotomy site influences postoperative pain following neurosurgical procedures: a retrospective study. Can J Anaesth 2007;54(7):544-548

19 Latz B, Mordhorst C, Kerz T, et al. Postoperative nausea and vomiting in patients after craniotomy: incidence and risk factors. J Neurosurg 2011;114(2):491-496

20 Kurita N, Kawaguchi M, Nakahashi K, et al. [Retrospective analysis of postoperative nausea and vomiting after craniotomy]. Masui 2004;53(2):150-155

21 Tisdall M, Crocker M, Watkiss J, Smith M. Disturbances of sodium in critically ill neurologic patients. J Neurosurg Anesthesiol 2006;18:57-63

22 Pauca AL, Savage RT, Simpson S, Roy RC. Effect of pethidine, fentanyl and morphine on post-operative shivering in man. Acta Anaesthesiol Scand 1984;28(2):138-143

23 Connolly ES, Rabinstein AA, Carhuapoma JR, Derdeyn CP, Dion J, Higashida Rt. Guidelines for the management of aneurysmal Subarachnoid haemorrhage: a guideline for healthcare professionals from the American Heart Association /American Stroke Association. Stroke 2012;43(6):1711-1737

24 Andrews PJ, Sinclair HL, Rodriguez A, et al; Eurotherm 3235 Trial Collaborators. Hypothermia for Intracranial Hypertension after Traumatic Brain Injury. N Engl J Med 2015;373(25):2403-2412

25 Cooper DJ, Nichol AD, Bailey M, et al. Effect of early sustained prophylactic hypothermia in neurologic outcomes among patients with severe traumatic brain injury. JAMA 2018;320(21):2211-2220

26 Li JX, Jin EZ, Yu LH, et al; Li Jing-Xui. Oral N-acetylcysteine for prophylaxis of contrast-induced nephropathy in patients following coronary angioplasty: A meta-analysis. Exp Ther Med 2017;14(2):1568-1576 